\title{
EL FUTURO DE LA APLICACIÓN DESCENTRALIZADA DE LA DEFENSA DE LA COMPETENCIA
}

\author{
Francisco Hernández Rodríguez
}

\begin{abstract}
Sumario: 1. Introducción 2. El nacimiento del Sistema DESCENTRALIZADO DE DEFENSA DE LA COMPETENCIA: VENTAJAS E INCONVENIENTES DEL MODELO ESPAÑOL 3. LOS MECANISMOS DE COORDINACIÓN Y EL SISTEMA DE REPARTO DE COMPETENCIAS PREVISTO EN LA LEY 1/2002 4. LA REALIDAD DE LAS AUTORIDADES AUTONÓMICAS DE DEFENSA DE LA COMPETENCIA 5. PRESUPUESTOS PARA UNA FUTURA REFORMA DEL SISTEMA DE APLICACIÓN DESCENTRALIZADA DE LA DEFENSA DE LA COMPETENCIA.
\end{abstract}

\section{INTRODUCCIÓN}

Transcurridos ya más de quince años desde que el Tribunal Constitucional atribuyese a las Comunidades Autónomas competencias ejecutivas en materia de defensa de la competencia, dando lugar a la aparición de diversas autoridades autonómicas de defensa de la competencia y a la creación de un sistema descentralizado sin parangón e otros países de nuestro entorno, disponemos ya de una experiencia que nos permite realizar un análisis realista sobre el funcionamiento del sistema.

La creación de autoridades de competencia autonómicas ha estado, desde el principio, rodeada de críticas y de voces que desde diversos ámbitos han venido proponiendo su supresión. Tras la desaparición de las autoridades de Castilla la Mancha y de Madrid, aunque esta última simplemente se 
convirtió en un órgano de instrucción, y la publicación del informe CORA, que proponía la supresión de los órganos autonómicos de competencia parecía que estábamos asistiendo a la desaparición del sistema descentralizado de defensa de la competencia pocos años después de su puesta en marcha. Sin embargo, en vez de suprimirlos, algunas Comunidades Autónomas optaron por reformar sus órganos de competencia con la intención de hacerlos más baratos y eficientes (Valencia, Castilla León), otras acometieron reformas más profundas (Galicia, Andalucía) y finalmente, un tercer grupo de Comunidades, que había reformado sus órganos de competencia hacía poco tiempo, los ha mantenido igual (Cataluña, País Vasco). Así las cosas, la realidad es que, con el paso de los años, el funcionamiento del sistema descentralizado ha ido adquiriendo una mayor eficacia y corrigiendo algunos de sus defectos, a la vez que se mejora la coordinación y las autoridades autonómicas reorientan su actividad hacia aquellas áreas en las que puede resultar más útil.

El objeto de este breve comentario no es más que realizar algunas reflexiones sobre el funcionamiento del sistema de aplicación descentralizado de defensa de la competencia y proponer algunas líneas de actuación que puedan mejorar su funcionamiento en el futuro.

\section{EL NACIMIENTO DEL SISTEMA DESCENTRALIZADO DE DEFENSA DE LA COMPETENCIA: VENTAJAS E INCONVENIENTES DEL MODELO ESPAÑOL}

La sentencia del Tribunal Constitucional 208/1999 de 11 de noviembre, al reconocer a las Comunidades Autónomas competencias ejecutivas en materia de defensa de la competencia abrió la puerta a la creación en España de un sistema descentralizado de aplicación de la normativa de defensa de la competencia.

El Tribunal Constitucional estimó el recurso interpuesto por la Generalitat de Cataluña y el Gobierno Vasco al entender que aquellas Comunidades Autónomas que tenían asumidas competencias en materia de comercio interior no podían ser privadas de la posibilidad de aplicar la Ley de defensa de la Competencia en sus respectivos territorios. Sin embargo, la legislación sobre defensa de la competencia, al ser considerada legislación mercantil quedaba reservada al Estado.

La aplicación descentralizada de la normativa de defensa de la competencia presenta, a priori, algunas ventajas, como que puede dar lugar a un incremento de la actividad en materia de defensa de la competencia, la mayor cercanía y el mejor conocimiento de los mercados locales por parte de las Autoridades autonómicas, o que permite a la Autoridad estatal focalizar sus recursos en los casos más graves. 


\section{Tabla 1. Aplicación de los mecanismos de asignación de expedientes en aplicación de la Ley 1/2002}

\begin{tabular}{|c|c|c|c|c|c|c|c|c|c|c|c|c|}
\hline & 写 & تَّ & 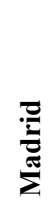 & $\frac{\pi}{\frac{\pi}{0}}$ & 莡 & 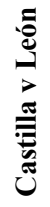 & 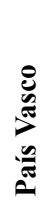 & & & 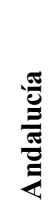 & Uू: & 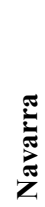 \\
\hline 2002 & 2 & & & & & & & & & & & \\
\hline 2003 & 15 & & & & & & & & & & & \\
\hline 2004 & 9 & & & & & & & & & & & \\
\hline 2005 & 11 & 1 & 3 & 2 & & & & & & & & \\
\hline 2006 & 12 & 8 & 6 & 2 & 4 & 1 & 4 & 1 & & & & \\
\hline 2007 & 8 & 11 & 6 & 3 & 7 & 8 & 10 & 0 & 1 & & & \\
\hline 2008 & 8 & 17 & 15 & 5 & 7 & 2 & 12 & 1 & 3 & 14 & 3 & \\
\hline 2009 & 11 & 7 & 9 & 9 & 2 & 7 & 7 & 1 & 4 & 14 & 2 & \\
\hline 2010 & 16 & 13 & 21 & 8 & 4 & 5 & 10 & 2 & 2 & 14 & 5 & \\
\hline 2011 & 14 & 14 & 15 & 7 & 9 & 3 & 12 & 2 & 3 & 15 & 7 & \\
\hline 2012 & 12 & 21 & 8 & 7 & 2 & 7 & 9 & 0 & 2 & 11 & 6 & \\
\hline 2013 & 7 & 8 & 16 & 12 & 5 & 13 & 7 & 4 & 3 & 22 & 8 & \\
\hline 2014 & 7 & 9 & 11 & 9 & 5 & 13 & 4 & 3 & 3 & 26 & 2 & 5 \\
\hline Total & 132 & 109 & 110 & 64 & 45 & 59 & 75 & 14 & 21 & 116 & 33 & 5 \\
\hline
\end{tabular}

Fuente: Web expedientes interna CNMC.

Por lo que respecta al incremento de actividad los datos corroboran que la creación de autoridades autonómicas ha supuesto un incremento muy notable del número de expedientes que se tramitan cada año. El aumento de actividad, tanto sancionadora como de promoción dará lugar a una mayor competencia en el mercado español y a un aumento de la productividad y de la eficiencia de las empresas. Así, en el año 2014 se sometieron al trámite de asignación 98 expedientes, de los que 74 se iniciaron en las autoridades autonómicas y 24 en la CNMC. Como pone de manifiesto la propia CNMC, «el mayor número de casos presentados en la respectiva $C A$, frente a los presentados en la CNMC, se debe, por un lado, al conocimiento cada vez mayor 
que tiene el mercado de las autoridades autonómicas y de sus competencias, así como a la actividad de oficio de éstas» ${ }^{1}$.

$\mathrm{Si}$ a los expedientes sometidos a asignación le añadimos las 36 colaboraciones no regladas que han tenido lugar en 2014 resulta que el volumen de casos relacionados de una u otra forma con las autoridades autonómicas es de 130. Al margen del sistema descentralizado se encuentran los casos que entran directamente en la CNMC y son resueltos por ésta sin pasar el trámite de asignación.

En segundo lugar, la mayor cercanía y el conocimiento más profundo de los mercados locales que pueden tener los órganos autonómicos es la ventaja más comúnmente reconocida de la aplicación descentralizada ${ }^{2}$. En los casos de dimensión local, la cercanía de la autoridad facilita la denuncia y permite obtener información de forma más rápida y con un menor coste. También se puede realizar el seguimiento de mercados locales de una forma más eficiente.

Otra ventaja ligada a la aplicación descentralizada de la normativa de defensa de la competencia es que la autoridad nacional puede concentrar sus esfuerzos en los grandes casos, aquellos que resultan más perjudiciales para la sociedad en su conjunto y que por su dimensión y complejidad requieren de importantes recursos. Así, por ejemplo, si nos fijamos en las resoluciones del extinto Tribunal de Defensa de la Competencia en una fecha anterior al comienzo de la descentralización como el año 1999, nos encontramos con resoluciones como «peleteros Utrera», «hoteles Hernani», «Colegio de ingenieros técnicos industriales de Burgos», «material eléctrico Murcia 2», o «funerarias Tenerife». En cambio, y quizá como consecuencia del elevado número de casos resueltos de naturaleza local, escaseaban los expedientes de dimensión nacional. En la actualidad, asuntos como los citados son resueltos por las autoridades autonómicas y si nos fijamos en los expedientes de la CNMC podemos comprobar que son mayoritariamente casos relacionados con grandes empresas que afectan a todo el mercado nacional.

Frente a estas ventajas, los detractores del sistema alegan que supone la duplicación de organismos y que puede romper la unidad de aplicación del derecho de la competencia en el territorio nacional. Evidentemente todo proceso de descentralización supone la creación de organismos autonómicos donde antes sólo había un órgano central. Sin embargo, una cosa es el aumento del número de organismos, que siempre tiene un coste que puede ser

${ }^{1} \mathrm{CNMC}$, «Aplicación de los mecanismos de asignación de expedientes CNMC/ CCAA y novedades», diciembre 2014.

2 En este sentido vid. MARCos, F, «Autoridades autonómicas de defensa de la competencia en vías de extinción», Documento de Trabajo serie Política de la Competencia, n³1/2002, Instituto Universitario de Estudios Europeos, pág. 6. 
rentable o no dependiendo de cómo funcione el nuevo sistema y, en este caso, del valor que se le de al incremento de la actividad de defensa de la competencia y otra muy diferente es que se pueda hablar de duplicidades. Existe duplicidad entre dos organismos cuando ambos pueden resolver el mismo caso dependiendo del lugar donde se presente la denuncia o de la voluntad del denunciante. Sin embargo, el sistema que crea la Ley de coordinación garantiza que sea cual sea el lugar de presentación de la denuncia, siempre será resuelta por el único organismo que resulte competente de acuerdo con los criterios de asignación que la propia Ley contiene. Es decir, existen diferentes organismos que aplican la misma normativa a casos diferentes en función de si sus efectos se limitan a la esfera autonómica o la superan alcanzando una dimensión nacional.

Por lo que respecta a la posibilidad de que se rompa la unidad de criterio en la aplicación de la Ley de Defensa de la Competencia, la Ley de coordinación contiene algunas medidas para evitar que esto se produzca y la realidad es que no se ha producido hasta la fecha. La Ley 1/2002 convierte a la CNMC en la guardiana del sistema al permitirle que se persone en todos los expedientes de las autoridades autonómicas.

\section{LOS MECANISMOS DE COORDINACIÓN Y EL SISTEMA DE REPARTO DE COMPETENCIAS PREVISTO EN LA LEY 1/2002}

Para llevar a la práctica lo establecido en la sentencia del Tribunal Constitucional resultaba imprescindible la aprobación de una Ley que estableciese los criterios para el reparto de competencias entre la Autoridad estatal y las autonómicas y crease los mecanismos de coordinación necesarios para garantizar una aplicación uniforme de la Ley de Defensa de la Competencia en todo el territorio nacional. Con este fin se aprobó la Ley 1/2002, de 21 de febrero de coordinación de las competencias del Estado y las Comunidades Autónomas en materia de defensa de la competencia. El contenido de la Ley se puede dividir en dos grandes bloques: el primero se refiere al reparto de competencias y el segundo a la coordinación entre Autoridades,

Por lo que respecta al reparto de competencias, (arts. 1-3), la Ley establece los puntos de conexión que delimitan el ejercicio de las competencias por parte del Estado y de las Comunidades Autónomas y crea un mecanismo para resolver los conflictos que pueda generar la aplicación de los puntos de conexión. El artículo primero acude al criterio de la supra-territorialidad como criterio de asignación de competencias al Estado, de forma que sólo corresponderán a las Comunidades Autónomas con competencias en la materia los procedimientos que sin afectar a un ámbito superior al de una Comunidad Autónoma o al conjunto del mercado nacional puedan alterar la libre competencia en el ámbito de la respectiva Comunidad Autónoma. 
Como acertadamente afirma MARCOS, el recurso a la supra-territorialidad como criterio de asignación de competencias al Estado desequilibra siempre la balanza del reparto de asuntos en beneficio del Estado y sienta las bases de posibles conflictos ${ }^{3}$. La experiencia de estos años nos ha enseñado que en un mercado único, regido por la libertad de establecimiento y la libre circulación de personas y mercancías en todo el territorio nacional, es difícil encontrar conductas, aún de pequeña dimensión, cuyos efectos no desborden de alguna forma el límite territorial de una Comunidad Autónoma. Un buen ejemplo de ello lo tenemos en el caso de las desmotadoras de algodón. Un caso en el que encontrándose en Andalucía todas las desmotadoras y más del $99 \%$ de la producción de algodón fue asignado a la CNMC porque se entendió que ese porcentaje menor del 1\% del algodón que se producía fuera de la Comunidad de Andalucía era suficiente para producir el desbordamiento.

Además, el artículo primero contempla una serie de casos en los que determinadas conductas, a pesar de que se realicen exclusivamente en el territorio de una Comunidad Autónoma, se asignan al Estado al entender el legislador que alteran la libre competencia en un ámbito supraautonómico. Sin embargo, se echa en falta un precepto en sentido contrario que permita asignar a las Comunidades Autónomas aquellos casos en que por ser muy reducidos los efectos que producen fuera de la Comunidad Autónoma apenas afectan al mercado nacional.

$\mathrm{Si}$ al desequilibrio que preside el sistema de reparto de competencias previsto en la Ley 1/2002 le unimos el hecho de que determinadas materias, como por ejemplo la aplicación de los artículos 101 y 102 del Tratado de Funcionamiento de la Unión Europea, el control de concentraciones o la elaboración de reglamentos de exención por categorías están reservadas en exclusiva al Estado, resulta fácil comprender las críticas que consideran que esta la Ley refleja un elevado nivel de desconfianza hacia las Comunidades Autónomas así como la intención de reservar a la autoridad estatal competencias cuya titularidad tras la sentencia del Tribunal Constitucional es, cuando menos, discutible 4 .

Con relación a los mecanismos de coordinación (art. 4-5), entendemos que el legislador está pensando más en crear mecanismos para que el órgano estatal garantice una aplicación uniforme de las normas de competencia que en dotar al sistema de instrumentos que permitan una coordinación eficaz entre todas la autoridades. Así la Ley contempla un único mecanismo de

3 Vid Marcos, F., ob. cit. pág.10

${ }^{4}$ En este sentido vid., entre otros CRUCELEgui, J. L., «Los modelos de aplicación descentralizada del derecho de la competencia en la UE y en el Estado Español», Cuadernos europeos de Deusto nº 38, 2007, págs.81-129, pág.126. 
coordinación, el Consejo de Defensa de la Competencia, que se ha mostrado poco operativo en la práctica. En cambio, medidas como la obligación que se impone a los órganos autonómicos de notificar a la CNMC todos los acuerdos y resoluciones adoptados, tanto en la fase de instrucción como de resolución, que pongan fin al procedimiento, la posibilidad de que la Dirección de investigación se persone como interesada en los procedimientos administrativos tramitados por los órganos autonómicos o la petición por parte de la CNMC al órgano autonómico de informe preceptivo no vinculante respecto de aquellas conductas que afectando a un ámbito supraautonómico o al conjunto del mercado nacional incidan de forma significativa en el territorio de la respectiva Comunidad Autónoma, sí han servido para garantizar un alto grado de uniformidad en la aplicación de la Ley de defensa de la Competencia en todo el territorio nacional.

\section{LA REALIDAD DE LAS AUTORIDADES AUTONÓMICAS DE DEFENSA DE LA COMPETENCIA}

No resulta fácil referirse de forma general a las autoridades autonómicas de defensa de la competencia debido a la diversidad existente, que ha sido una característica del sistema descentralizado desde su nacimiento. Así, entre las autoridades existentes en la actualidad nos encontramos con realidades muy diversas tanto en lo que se refiere a su configuración jurídica como a su presupuesto y objetivos.

Se pueden distinguir tres fases en la evolución de las autoridades autonómicas de la Competencia que, en cierto modo, han seguido la estela que dejaba la normativa estatal en su evolución. En una primera fase, las autoridades autonómicas replicaban de forma mimética la naturaleza del Tribunal de defensa de la Competencia separando resolución e instrucción en órganos separados y denominándose tribunales. En la segunda fase, tras la creación de la Comisión Nacional de la Competencia algunas autoridades se reformaron para integrar instrucción y resolución en un órgano único siguiendo el modelo de la CNC. En la actualidad, tras la conversión de la Comisión Nacional de la Competencia en un órgano multifuncional, tras su integración con los reguladores sectoriales en el seno de la nueva Comisión Nacional de los Mercados y la Competencia se comienzan a gestar también autoridades autonómicas de tercera generación. Así, en el caso de Galicia está prevista la integración del Consello Galego da Competencia con el Instituto Gallego de Consumo para crear una autoridad única de competencia y consumo, mientras que en Andalucía se ha dotado de nuevas funciones a la Agencia Andaluza de Defensa de la Competencia.

A día de hoy hay en España 12 autoridades autonómicas de competencia que podemos clasificar en tres grupos: 
1. Autoridades con órgano de instrucción y resolución integrado: Andalucía, Cataluña, Galicia y País Vasco

2. Autoridades con una estructura dual de instrucción y resolución: Comunidad Valenciana, Castilla León, Aragón y Extremadura

3. Autoridades que sólo tienen órgano de instrucción (resuelve el Consejo de la CNMC): Canarias, Madrid, Murcia y Navarra.

Con independencia de su diversa configuración jurídica, desde un punto de vista práctico, la principal distinción entre autoridades autonómicas viene dada por las enormes diferencias existentes entre sus presupuestos y medios humanos y materiales, que determinan los niveles de actividad de cada una de ellas.

Si en un principio se podría pensar que la coordinación entre doce autoridades diferentes con la CNMC resultaría problemática, fundamentalmente por los defectos ya reseñados de la Ley de coordinación, la realidad ha sido muy distinta y el funcionamiento del sistema descentralizado ha funcionado con una mínima conflictividad y elevados niveles de cooperación. Así, desde su creación en 2002, los órganos de competencia sólo han acudido 4 veces a la junta consultiva en materia de conflictos y si bien el Consejo de Defensa de la Competencia, que es el principal órgano de coordinación creado por la Ley $1 / 2002$ ha resultado ser poco eficaz, se han creado diversos grupos de trabajo que funcionan con regularidad y contribuyen de forma notable a mantener la coordinación en la aplicación de la normativa de competencia, a la vez que permiten un intercambio fluido de información entre las distintas autoridades.

En el ámbito de la instrucción funcionan los grupos de trabajo de «aplicación de la Ley 1/2002 por los órganos de instrucción», «asignación de expedientes» $\mathrm{y}$ 《red de autoridades de competencia», que están presididos por la dirección de competencia de la CNMC. Además hay un grupo de trabajo de promoción y se está poniendo en marcha un grupo de trabajo de órganos de resolución. Uno de los mejores ejemplos del alto nivel de coordinación alcanzado es el funcionamiento de la red de competencia (REC).

Uno de los instrumentos de cooperación y coordinación entre las autoridades españolas de competencia que ha venido resultando más eficaz es la denominada «Red de Cooperación de los Órganos Competentes en materia de competencia $(R E C)$ ». Se trata de una red informática articulada en el marco de la plataforma de la administración electrónica, de la Administración General del Estado (AGE), gestionada por el Centro de Recursos de Comunicación e Información para Administraciones, Empresas y Ciudadanos (CIRCAB) 5 .

${ }^{5}$ La administración electrónica de la AGE se apoya en Portal de la Administración Electrónica en la dirección http://administracionelectronica.gob.es/ gestionada técnica- 
Se trata, como señala la propia $\mathrm{CNC}^{6}$, de «un espacio común de intercambio de información que permite la comunicación y coordinación fluida y permanente entre todas las autoridades de competencia de las distintas Administraciones (Estatal y autonómica)».

Para la actualización de este «espacio común de intercambio de información» se creó y está en funcionamiento desde $2008^{7}$ un Grupo de Trabajo, de los creados en el seno del Consejo de Defensa de la Competencia.

Esta Red permite, tanto la transmisión horizontal de información entre las autoridades de competencia españolas, como agilizar el funcionamiento del mecanismo de asignación de casos (en este caso con carácter bilateral entre la CNMC y la autoridad de competencia autonómica directamente afectada), así como la notificación interna entre la CNMC y las autoridades de competencia y de estas con aquella ${ }^{8}$, de forma rápida, segura y sin coste.

Otra manifestación de este clima de entendimiento son los cursos de inspección para personal de las autoridades autonómicas que organiza anualmente la CNMC o las jornadas nacionales de la competencia que se vienen celebrando desde el año 2008.

Por lo que se refiere a los niveles globales de actividad podemos aportar algunas cifras que demuestran que la descentralización en la aplicación del la normativa de competencia ha supuesto un aumento de actividad en términos generales. Así, desde 2001, año en que comienza a funcionar la Autoridad Catalana hasta diciembre de 2014 se sometieron 799 expedientes al trámite de asignación previsto en la Ley 1/2002. Esta cifra recoge sólo los expedientes que tuvieron entrada en una Autoridad Autonómica y las que entraron por la Comisión Nacional pero tenían, por lo menos en apariencia, dimensión autonómica. Los casos que entran por la Autoridad estatal y que, en opinión

mente por el CIRCABC (Centro de Recursos de Comunicación e Información para Administraciones, Empresas y Ciudadanos).

${ }^{6} \mathrm{CNC}$, Aplicación de la Ley 1/2002, de 21 de febrero, de coordinación de las competencias del Estado y las Comunidades Autónomas en materia de defensa de la Competencia 2008-2012, p. 41. http://www.cncompetencia.es/Portals/0/PDFs/Docs/CNC-informe\%20autonom\%C3\%ADas5_indexado.pdf

7 En la reunión del Consejo de Defensa de la Competencia, de 19 de diciembre de 2008 se creó el «Grupo de actualización y mantenimiento de la Red de Cooperación de los Órganos Competentes en materia de competencia». CNC, Aplicación de la Ley 1/2002..., op. cit. p. 41.

${ }^{8}$ Para analizar la experiencia en la asignación de casos la CNMC, como antes la $\mathrm{CNC}$, han publicado diversos informes específicos. El últimio de ellos estos informes se ha publicado en diciembre de 2014: CNMC, Aplicación de los mecanismos de asignación de expedientes CNMC/CCA y novedades Año 2014. Este informe se puede consultar en la web oficial de la CNMC en la dirección: http://www.cnmc.es/Portals/0/Ficheros/Competencia/Instituciones/201402_InformeCoordinacionCCAA.pdf 
de ésta, no limitan sus efectos exclusivamente at territorio de una Comunidad Autónoma no se someten al trámite de asignación y quedan, de facto, al margen del sistema previsto en la Ley 1/2002. Aunque la Ley de Coordinación no exceptúa estos expedientes del trámite de asignación, la CNC y ahora la CNMC lo han venido haciendo a pesar de la opinión contraria de las autoridades autonómicas.

Desafiando las predicciones más pesimistas, los hechos parecen corroborar que la aplicación descentralizada se encuentra en una fase de consolidación, caracterizada por un nivel de actividad en materia de defensa de la competencia cada vez más elevado, un alto grado de coordinación y cooperación entre las Autoridades, que han desarrollado mecanismos más eficaces que los inicialmente previstos en la Ley de coordinación, y muy baja conflictividad. Es el momento, por tanto, de superar ciertos temores y desconfianzas que han lastrado el funcionamiento del sistema y de crear un nuevo marco jurídico que sustituya al previsto en la Ley $1 / 2002$, permitiendo que la aplicación descentralizada suponga un aumento cuantitativo y cualitativo de la actividad relacionada con la defensa de la competencia en España en beneficio de toda la sociedad. Buena prueba de que avanzamos hacia una nueva etapa lo constituye el Plan Estratégico de la Comisión Nacional de los Mercados y la Competencia, que contiene interesantes propuestas para reforzar la colaboración con las autoridades autonómicas.

\section{PRESUPUESTOS PARA UNA FUTURA REFORMA DEL SISTEMA DE APLICACIÓN DESCENTRALIZADA DE LA DEFENSA DE LA COMPETENCIA}

Cualquier iniciativa de reforma del sistema de aplicación descentralizada de la defensa de la competencia debería asentarse sobre dos pilares fundamentales: por un lado, resulta necesario reformar la Ley 1/2002 de coordinación de competencias, una norma más orientada a resolver los conflictos, que en muchos casos generaría ella misma, fundamentalmente por el inadecuado sistema de reparto de competencias, que a sentar las bases de funcionamiento de un sistema eficiente basado en la lealtad constitucional y sin apenas conflictividad. Por otro lado, las autoridades autonómicas deben evolucionar a la luz de la experiencia adquirida y perfilar su actividad hacia aquellos ámbitos en los que su trabajo puede ser más eficaz, orientándose hacia aquellos sectores a los que a la CNMC le puede resultar más difícil llegar. Las recurrentes críticas a la duplicidad de organismos se responden con eficacia y complementariedad.

Por lo que respecta a los cambios normativos, hay que decir que si bien la reforma de la Ley de coordinación constituye el paso imprescindible para la mejora del sistema, no es ésta la única norma en la que se pone de manifiesto 
una cierta desconfianza hacia las Autoridades autonómicas. Así, nos parece significativo que en la Ley de Unidad de Mercado se asignen importantes competencias a la CNMC y sin embargo se prescinda totalmente de las autoridades autonómicas a pesar de que podrían complementar perfectamente la actividad de ésta. Si el cumplimiento de todas las funciones que la Ley de unidad de mercado atribuye a la CNMC puede resultar excesiva, las autoridades autonómicas están especialmente bien situadas para asumir ciertas funciones, por ejemplo, en relación con las corporaciones locales, incluso limitando su actividad a los ayuntamientos que no lleguen a un determinado número de habitantes, que en muchos casos se quedarán fuera del radio de actividad de la autoridad estatal. Las Autoridades autonómicas de competencia son organismos que, en sus respectivas comunidades, aplican normativa estatal y garantizan su cumplimiento, colaborando de esta forma a la unidad de mercado.

El modelo diseñado en la Ley $1 / 2002$, de 21 de febrero, de Coordinación de las Competencias del Estado y las Comunidades Autónomas en materia de Defensa de la Competencia ${ }^{9}$, para la asignación de casos, basado en «competencias excluyentes» (Estado/Comunidad Autónoma), contrasta notablemente con el diseñado en la Unión Europea sobre la base del Reglamento (CE) $\mathrm{n}^{\circ}$ 1/2003, del Consejo de 16 de diciembre de 2002, relativo a la aplicación de las normas sobre competencia previstas en los artículos 81 y 82 [hoy 101 y 102 TFUE] del Tratado ${ }^{10}$ y sus normas de desarrollo y complemento ${ }^{11}$.

Entendemos que este modelo, que no ha sido modificado tras la aprobación de la vigente $\mathrm{LDC}$, ni tras la creación de la CNMC, desaprovecha la

9 A este respecto, vid. Hernández Rodríguez, F., «La Ley 1/2002, de coordinación de las competencias del Estado y las Comunidades Autónomas en materia de defensa de la competencia», en Actas de derecho industrial y derecho de autor, ISSN 1139-3289, Tomo 22, 2001, págs. 1449-1457.

${ }^{10}$ DO L 1, de 4.1.2003, p. 1.

${ }^{11}$ Cfr. la Declaración común del Consejo y de la Comisión sobre el funcionamiento de la Red de Autoridades de Competencia (Esta Declaración común se puede descargar en http://ec.europa.eu/competition/ecn/joint_statement_es.pdf) y Comunicación de la Comisión sobre la cooperación en la Red de Autoridades de Competencia (DO C 101, de 27.4.2004, p. 43). Como recuerdan Ortiz Blanco, Luis y Lamadrid de Pablo, Alfonso («EU Competition Law Enforcement: Elements for a discussion on effectiveness and uniformity», en International Antitrust Law \& Policy: Fordham Competition Law 2011, Chapter 4, 2012, pp. 45 a 104, en concreto, 100, nota 222), a los que cita RodRíGUEz MIGUEZ, J. A. («La «Red Española de Autoridades de la Competencia»: las posibilidades de un modelo descentralizado para ganar en eficiencia», en esta misma publicación), este documento no sólo vincula a la Comisión, sino también a los Estados miembros que suscribieron, como se recoge en la propia Comunicación (nota 4), la Declaración común del Consejo y de la Comisión sobre el funcionamiento de la Red de Autoridades de Competencia, disponible en el registro del Consejo, en http://register.consilium.eu.int (documento no $15435 / 02$ ADD 1 ). 
oportunidad de flexibilizar el rígido sistema de asignación de casos previsto en aquélla ${ }^{12}$, ignorando que el modelo de la Unión Europea debe, en buena medida, su éxito al papel desempeñado por la European Competition Network (ECN), en la aplicación del principio básico de las competencias concurrentes, diseñado y aplicado en el marco de la Unión Europea ${ }^{13}$.

La asignación de casos entre la Comisión Europea y/o una o varias ANC no responde a criterios rígidos, sino que es aplicado de manera flexible, lo que contrasta acentuadamente con lo que ocurre en el ámbito del Derecho español de la competencia que, tal y como ha sido interpretado por nuestro Tribunal Constitucional, se asienta en un sistema de «competencias excluyentes», que se aplica atendiendo a criterios en exceso formalistas y, a la postre, mucho menos claros y eficientes ${ }^{14}$.

El debate se suscita así en torno a la expresión, nada ambigua y enormemente flexible ${ }^{15}$, de la Autoridad «mejor situada», si bien esta expre-

12 No obstante debe reconocerse, como apunta RodRíGuez Miguez, J. A. «que la LDC corrigió uno de los rasgos más evidentes de la Ley 1/2002 que era la existencia de notables asimetrías entre las exigencias impuestas al, por aquel entonces SDC, y las requeridas a los órganos autonómicos en materia de competencia. A través de la reforma del Artículo 5.Dos, letra b), de la Ley 1/2002, introducida por la LDC, estos desequilibrios son corregidos de forma sencilla, superando el absurdo de que las Comunidades Autónomas hubieran de mandar copia de todas las denuncias y el SDC únicamente nota sucinta.

${ }_{13}$ No se trata, sin embargo, del criterio general en el ámbito de la competencia pues, por ejemplo, en materia de Ayudas de Estado, donde la Comisión tiene competencias exclusivas o en sede de concentraciones la Comisión o las ANC «tendrá(n) competencia exclusiva para adoptar las decisiones que correspondan, conforme al Reglamento (CE) $\mathrm{n}^{\circ}$ 139/2004 del Consejo, de 20 de enero de 2004, sobre el control de las concentraciones entre empresas (DO L 24, de 29.01.2004, p. 1), cuando se aprecie que tienen «dimensión comunitaria» o solamente nacional, lo que se hace en función de parámetros concretos y fijos previstos en la propia norma. En materia de concentraciones resulta de interés la STPI sentencia de 14 de julio de 2006, Endesa/Comisión, asunto T-417/05, Rec. 2006 p. II-2533, a propósito de la frustada operación Gas Natural/ENDESA.

${ }^{14}$ En el punto 5 de la Comunicación de la Comisión sobre la cooperación en la Red de Autoridades de Competencia (DO C 101, de 27.4.2004, p. 43) se señala «5. El Reglamento [1/2003] del Consejo se basa en un sistema de competencias concurrentes en el que todas las autoridades de competencia tienen potestad para aplicar los artículos 81 y 82 del Tratado y deben buscar un reparto eficaz de los asuntos cuando se considere que una investigación es necesaria. [...]»

${ }_{15}$ Como recuerda Folguera, el Reglamento 1/2003 no establece normas que regulen con carácter imperativo el reparto de asuntos entre la Comisión y las Autoridades nacionales de la competencia, sino que opta porque éste se decida de forma consensuda y flexible en el seno de la propia REC (ECN) (Folguera CRESPO, J.: «El concepto de autoridad bien situada y la cooperación en el seno de la red de autoridades de competencia», en Martínez Lage, S./Petitbó, J. A. (Dirs.): La Modernización del Derecho de la Competencia en España y la Unión Europea, Fundación Rafael del Pino, Madrid, 2005, pp. 
sión se reserva, en realidad, para cuando la atribución la pretenda la propia Comisión ${ }^{16}$.

En este sentido, y como ya tuvimos ocasión de señalar, parecería más razonable admitir una aplicación más «flexible» de este criterio, de tal suerte que la simple existencia de «desbordamientos» del ámbito territorial autonómico; esto es, de intercambios comerciales con otras zonas del territorio nacional, o la existencia de algún establecimiento fuera de la comunidad autónoma de referencia, no debería generar, por sí solo, una alteración de la libre competencia en un ámbito supra-autonómico en el sentido del artículo 1 de la Ley 1/2002.

Por tanto, nos parece razonable interpretar, como señala Rodríguez Miguez $^{17}$, esta norma en el sentido de que la competencia ejecutiva corresponderá al Estado sólo cuando la conducta restrictiva afecte de forma significativa a un mercado supra-autonómico o al conjunto del mercado nacional.

La vía sería admitir que el desbordamiento debería ser sensible o significativo, concepto ya presente en el ordenamiento español en textos como la Ley 27/1999, de 16 de julio, de cooperativas, que resulta de aplicación a las sociedades cooperativas que desarrollen su actividad cooperativizada en el «territorio de varias Comunidades Autónomas», excepto cuando en una de ellas se desenvuelva «con carácter principal», caso en el que la cooperativa quedaría sometida a la legislación específica de la correspondiente Comunidad Autónoma ${ }^{18}$.

209 a 226; en concreto, p. 214. Respecto de los casos de cártel en que se apreció afectación del mercado intracomunitario examinados por la CNC desde su creación y en los que se consideró que ésta era la Autoridad mejor situada, vid. CORTI VARELA, J.: «Tipología de cárteles un estudio de los 20 casos resueltos por la CNC y la CNMC», en el Capítulo 3 de la presente obra.

${ }^{16}$ Comunicación ECN, punto 8 in fine: «9. Estos criterios indican que debe existir un vínculo material entre la infracción y el territorio de un Estado miembro para que se considere bien situada a la autoridad de competencia de dicho Estado. Puede esperarse que, en la mayoría de los casos, las autoridades de los Estados miembros donde la competencia se ve afectada apreciablemente por una infracción estarán bien situadas, siempre que puedan poner fin efectivamente a la infracción, a través de acciones individuales o concurrentes, a menos que la Comisión esté mejor situada (véanse los puntos 14 y 15). El subrayado es nuestro.

17 Rodríguez Miguez, J.A., «La "Red Española de Autoridades de la Competencia”: las posibilidades de un modelo descentralizado para ganar en eficiencia», op. cit.).

18 Esta interpretación ha sido defendida por COSTAS COMESAÑA, J.: «La descentralización del Derecho español de defensa de la competencia: Una visión crítica de la STC 308/99 y la Ley de coordinación», en el marco del Encuentro sobre el presente y el futuro del sistema comunitario y español de defensa de la competencia, organizado por la Universidad Internacional Menéndez Pelayo, sede de Galicia, celebrado en Pontevedra, los días 12 a 14 de julio de 2006, de quien tomamos esta idea y que consideramos plenamente vigente y asumible. 
Esta idea se podría combinar con la atribución de una competencia general a la CNMC que podría asumir la competencia de aquellos casos autonómicos cuya importancia o especial complejidad podría superar la capacidad de actuación de la correspondiente autoridad autonómica.

Otra fórmula podría ser, como sugiere Berasategui ${ }^{19}$, tomar como punto de partida la Ley de la Competencia alemana, estableciendo como único punto de conexión el efecto territorial de la conducta (artículo 48.2) pero con un mecanismo de flexibilidad: que permite a la autoridad regional ceder un asunto a la autoridad central (artículo 49.3), y el caso inverso, si ninguna otra autoridad regional se opone (artículo 49.4). Asimismo, y como nos recuerda este mismo autor, los tribunales alemanes exigen que el efecto sea 'sustancial'; término equivalente al de afectación sensible o significativa al que ya aludimos.

Para ello sería preciso modificar la Ley $1 / 2002$, circunstancia que podría aprovecharse para incorporar la nueva redacción a la vigente LDC, aprovechando los huecos que en su articulado ha dejado la aprobación de la Ley 3/2013, de 4 de junio, de creación de la Comisión Nacional de los Mercados y la Competencia. Esta solución técnica implicaría derogar la actual Ley 1/2002 y dotar de pleno contenido a la LDC, al incorporar una cuestión de tanta transcendencia como la relativa al reparto competencias.

El segundo gran tema sería crear una verdadera Red Española de Autoridades de Competencia. En este punto el sistema español es más avanzado que el europeo, aunque sus resultados han demostrado que podría ser más eficiente. El punto de partida sería el Consejo de Defensa de la Competencia, órgano del que carece propiamente la ECN.

Sería necesario reformularlo, tanto en su composición, artificialmente paritaria y con una presidencia ex lege, como en sus funciones y sacar mayor partido a la red virtual CIRCAB, cuya dimensión pública debería potenciarse y abrirse al público del mismo modo que lo está la ICN o la ECN. Dando así la idea de un verdadero sistema integrado de autoridades de competencia españolas que, desde sus respectivas competencias, trabajan en un objetivo común: el fortalecimiento de la competencia en el mercado español.

Esta idea debe presidir también la intervención de la CNMC en los expedientes autonómicos, reemplazando, posiblemente, su sistemática personación como parte interesada por una legitimación amplia para acudir a la vía contenciosa, en caso de desacuerdo y por un sistema de Amiicus Curiae, como el vigente en las relaciones entre la Comisión Europea y las Autorida-

19 En este sentido, Berasategi, J., «Defensa de la competencia descentralizada», Diario Cinco Días, del 19-10-2006. Este breve, pero interesante artículo, cuya contenido desconocemos si ha publicado con mayor detalle en algún otro sitio, puede descargarse en http://cincodias.com/cincodias/2006/10/19/economia/1161370547_850215.html 
des nacionales de competencia, respecto de los expedientes en los que ésta última es la autoridad «mejor situada».

Por lo que respecta a la actividad de las autoridades autonómicas, la experiencia demuestra que la promoción es uno de los campos en los que su actividad puede resultar más eficaz y complementaria de la de la Comisión Nacional.

No existe en nuestro Derecho una definición de «promoción de la competencia», sino una serie de ámbitos, metodologías y funciones que contribuyen a evitar o suprimir comportamientos anticompetitivos ${ }^{20}$.

Tal y como ha puesto de manifiesto la Comisión europea en su Comunicación «una política de competencia proactiva para una Europa competitiva» ${ }^{21}$, la promoción de la competencia no sólo supone la realización de actividades de difusión entre los operadores económicos y las propias Administraciones Públicas acerca de cuáles son las conductas contrarias a la competencia y las ventajas para la sociedad de disponer de mercados más competitivos. La moderna dimensión de la promoción de la competencia incluye además todas aquellas otras acciones destinadas a impulsar o, en su caso, eliminar del Ordenamiento jurídico aquellas normas o actuaciones que restrinjan de manera innecesaria o desproporcionada la competencia.

Por lo tanto, su objetivo no son sólo los operadores económicos, públicos o privados, sino y de manera muy singular, como ya hemos anticipado, las Administraciones Públicas, en su dimensión de Poder Público, cuyas actuaciones, decisiones o reglamentaciones pueden impedir o restringir el funcionamiento competitivo de los mercados.

Un intento de definir de manera sistemática la Promoción de la competencia ${ }^{22}$ lo encontramos en la que ofrece el Advocacy Working Group, de la Internacional Competition Network, que en $2002^{23}$ ofrece la siguiente definición:

"Conjunto de actividades de la autoridad de la competencia encaminadas a promover un entorno competitivo para el desarrollo de las actividades económicas, por medio de mecanismos no coactivos, sobre todos a

${ }^{20}$ En este sentido, vid. Rodríguez Miguez, J. A.: «Promoción versus defensa de la Competencia en España: una visión de conjunto», Revista Dereito, de la Facultad de Derecho de la Universidad de Santiago de Compostela, $\mathrm{n}^{\circ}$ 19, 2, 2010, pp. 51 a 98, p. 56.

${ }^{21}$ COMUNICACIÓN DE LA COMISIÓN Una política de competencia proactiva para una Europa competitiva. COM (2004) 293 final.

${ }_{22}$ Como veremos más adelante, la Exposición de Motivos de la Ley 1/2009, del 12 de febrero, de la Autoridad Catalana de la Competencia (BOE núm. 74, de 27 de marzo de 2009), párr. 7.

${ }^{23}$ Advocacy and Competition Policy. Report prepared by the Advocacy Working Group ICN's Conference. Naples, Italy, 2002, p. 115. En particular, p. 25. 
través de la interacción con otras administraciones públicas, la interacción con agentes económicos y la sensibilización del público acerca de los beneficios de la competencia ${ }^{24} \gg$.

Dada la importancia que esta función tiene para las Autoridades autónómicas de competencia, no debe extrañar que una de las definiciones más completa y descritiva de la misma sea la que ofrece la E. de M. de la Ley 1/2009, del 12 de febrero, de la Autoridad Catalana de la Competencia ${ }^{25}$, que establece que «La promoción de la competencia incluye, además de la elaboración, canalización y divulgación de estudios y trabajos relacionados con la competencia, la capacidad de elaborar informes desde el punto de vista de la competencia, en el momento procedimental oportuno, tanto de los proyectos normativos de las administraciones públicas de Cataluña como de sus actuaciones, si afectan o pueden afectar, directa o indirectamente, al funcionamiento competitivo de los mercados.»

De hecho, esta función figura de manera habitual en las normas reguladoras de las autoridades autonómicas de competencia y en alguna incluso aparece en el título ${ }^{26}$.

Aunque su incorporación como función propia de las Autoridades de Competencia en España se produjo ya con la Ley 16/1989, de 16 de julio, de Defensa de la Competencia, que incorporaba alguno de los instrumentos de promoción de la competencia, lo cierto es que es a partir de la aprobación de la actual Ley 15/2007, de 3 de julio, también denominada como la anterior «de Defensa de la Competencia», cuando esta función cobra verdadera carta de naturaleza en nuestro país, siguiendo las recomendaciones del Libro blanco para la reforma del sistema español de defensa de la competencia, que elaborado por el Ministerio de Economía y Hacienda y presentado el 20 de enero de 2005, sirvió de punto de partida para la reforma de la competencia es España ${ }^{27}$.

${ }^{24}$ La traducción la tomamos de la ponencia de D. LuIs BERENGUER, «¿Qué se entiende por promoción de la competencia», ya citada. El texto original reza del siguiente modo: "Competition advocacy refers to those activities conducted by the competition authority related to the promotion of a competitive environment for economic activities by means of non-enforcement mechanisms, mainly through its relationships with other governmental entities and by increasing public awareness of the benefits of competition.»

25 BOE núm. 74, de 27 de marzo de 2009.

${ }^{26}$ Cfr. Ley 6/2007, de 26 de junio, se denomina, de manera muy acertada, «de Promoción y Defensa de la Competencia de Andalucía» (BOJA núm. 131, de 4 de julio). Paradójicamente, como señala RodRÍGUEZ Miguez («Promoción versus defensa ..., op. cit., p. 33), el órgano andaluz de competencia recupera en exclusiva la expresión «defensa», pues se denomina «Agencia de Defensa de la Competencia de Andalucía», siguiendo la práctica tradicional de otros órganos españoles en la materia.

${ }^{27}$ El Libro blanco para la reforma del sistema español de defensa de la competencia dedicaba un epígrafe específico, bajo el título «Promoción de la defensa de la competen- 
De hecho, dicho documento planteaba expresamente la necesidad de un «refuerzo de las funciones de la Comisión Nacional de Defensa de la Competencia en el ámbito del control normativo, de forma que disponga de información y de instrumentos para tratar de minimizar las distorsiones introducidas por el propio sector público.»

Pues bien, entendemos que son las autoridades autonómicas quienes están mejor situadas para desarrollar algunas de las funciones de promoción cuya realización preocupaba a los redactores del «Libro Blanco». En este sentido, las autoridades autonómicas deben reforzar su actividad en materia de control normativo en su Comunidad, centrándose no sólo en su gobierno sino también en los Ayuntamientos, donde a la CNMC le costaría mucho más llegar. Esta labor de control normativo debe ir más allá de la mera elaboración de informes y llegar hasta la impugnación de normas, incluyendo aquí las ordenanzas municipales, tal y como están haciendo ya algunas autoridades.

Esta labor entronca directamente con la actividad de las autoridades autonómicas como garantes de la unidad de mercado, como prueba el hecho de que algunas de ellas hayan sido designadas directamente como puntos de conexión en aplicación de lo dispuesto en la Ley de Unidad de Mercado y otras colaboren informando los diversos expedientes que afectan a su Comunidad. A pesar del «olvido» voluntario o involuntario del legislador, que da lugar a que no aparezcan mencionadas en la Ley de Unidad de Mercado, en la práctica las autoridades autonómicas se están convirtiendo en una pieza importante del sistema.

Finalmente, y sin salir del ámbito de la promoción, otro instrumento que se ha mostrado tremendamente eficaz son los informes sectoriales. A diferencia de la CNMC, que hace informes de promoción sobre problemas de competencia de dimensión nacional, las autoridades autonómicas pueden dotar a sus informes de una dimensión local, complementando en este sentido la actividad de la dirección de promoción de la CNMC. Un buen ejemplo de ello lo constituye el informe del Consello Galego da Competencia sobre «el mercado de los carburantes de automoción en Galicia: una visión desde la libre competencia ${ }^{28}$. Galicia es una de las Comunidades Autónomas con los carburantes más caros de España, y ninguno de los diversos informes elabo-

cia» dentro del apartado general de «Proyección social de la defensa de la competencia» a esta materia (apartados 211 a 214.

${ }^{28}$ El informe se puede consultar en la página web del Consello Galego da Competencia www.consellogalegodacompetencia.es. Otros informes realizados por este organismo que también se pueden consultar en su web son los referidos a la problemática de los precios en el sector lácteo gallego, los aparcamientos de los aeropuertos gallegos desde el punto de vista de la competencia o el informe sobre uniformes escolares. 
rados sobre el sector tanto por la Comisión Nacional de la Energía como por la Comisión Nacional de la Competencia explicaban las razones de este hecho. Por esta razón, el Consello Galego da Competencia publicó un informe en el que consideraba que el diferencial de precios de Galicia con relación a otras Comunidades se debía, por lo menos en parte, a la existencia de una menor competencia en el mercado minorista y al menor número de gasolineras «low cost», especialmente de hipermercados, existente en la Comunidad. Las recomendaciones del informe para incrementar la competencia en el mercado minorista de carburantes de automoción en Galicia fueron asumidas por la Xunta de Galicia que, entre otras medidas, acaba de aprobar un Decreto para facilitar la apertura de gasolineras en la Comunidad, en los términos propuestos en el informe ${ }^{29}$. Actuaciones de este tipo, que muy difícilmente puede realizar una autoridad estatal, dotan de sentido a las autoridades autonómicas y constituyen una línea a seguir.

Sólo el trabajo conjunto de la autoridad nacional y las autonómicas ha permitido pasar del panorama de desconfianza y conflictividad que dejaba entrever la Ley 1/2002 de coordinación de las competencias del Estado y las Comunidades Autónomas en materia de defensa de la competencia, hasta la realidad actual, en la que el sistema descentralizado funciona con relativa eficacia en un marco de colaboración y baja conflictividad. Si como afirmaba Erhard en su obra «Prosperity through competition», la libre competencia es la mejor forma de alcanzar y garantizar la «prosperidad» ${ }^{30}$, no cabe duda de que un sistema que permite destinar más recursos a la defensa de la competencia y sobre todo, que permite que la labor de las autoridades de competencia llegue a todos los rincones, empresas y ciudadanos de un país, redunda en beneficio de toda la sociedad.

TITTLE: The future of the decentralized application of defense of competition.

RESUMEN: El objetivo de estas breves líneas no es más que realizar algunas reflexiones sobre el funcionamiento del sistema de aplicación descentralizado de defensa de la competencia y proponer algunas líneas de actuación que puedan mejorar su funcionamiento en el futuro.

${ }_{29}$ Decreto 45/2015 de 26 de marzo, por el que se regula el procedimiento integrado para la implantación de instalaciones de distribución al por menor de productos petrolíferos, su puesta en funcionamiento, y se determinan los órganos competentes para el ejercicio de la potestad sancionadora en materia de hidrocarburos. Diario Oficial de Galicia, $\mathrm{n}^{\circ} 68,23$ de abril de 2015, págs. 13992 y ss.

30 ERHARD, L., «Prosperity through competition», edición inglesa, New York 1958, pág.1. 
Así, de un lado, la aplicación descentralizada de la normativa de defensa de la competencia presenta, a priori, algunas ventajas, como que puede dar lugar a un incremento de la actividad en materia de defensa de la competencia, la mayor cercanía y el mejor conocimiento de los mercados locales por parte de las Autoridades autonómicas, o que permite a la Autoridad estatal focalizar sus recursos en los casos más graves.

Sin embargo, de otro lado, los detractores del sistema descentralizado alegan que supone la duplicación de organismos y que puede romper la unidad de aplicación del derecho de la competencia en el territorio nacional.

En otro orden de consideraciones, será menester de estas líneas ilustrar brevemente cuál ha sido el iter protagonizado por las autoridades de competencia, auspiciado por modificaciones normativas a las que se han ido adaptando las distintas autoridades de competencia. Sin duda, en todo ese recorrido se alza como piedra angular la promoción de la competencia, cuestión que suscita la preocupación y ocupación tanto del legislativo cuando dicta la Ley 1/2002, de 21 de febrero de coordinación de las competencias del Estado y las Comunidades Autónomas en materia de defensa de la competencia como de las propias autoridades que apuestan por la sinergia con instrumentos de cooperación tan eficientes como la «Red de Cooperación de los Órganos Competentes en materia de competencia (REC)».

PALABRAS CLAVE: sistema descentralizado, promoción de competencia, autoridades de competencia, ventajas, inconvenientes.

ABSTRACT: The aim of these brief lines is not any more that to realize any reflections on the functioning of the system of application decentralized of defense of competition and to propose some lines of action that could improve its functioning in the future.

Thus, on one hand, the decentralized application of the regulation of defense of competence presents, a priori, some advantages, as which it can give place to an increase of the activity in defense of competence matter, the major nearness and the best knowledge of the local markets on the part of the autonomous Authorities, or that allows to the state Authority to focus its resources in the most serious cases.

Nevertheless, on the other hand, the detractors of the decentralized system invoke that it supposes the duplication of organisms and that it can break the unit of application of the law of competence in the national territory.

In another order of considerations, it will been necessary of these lines to illustrate brief which has been the iter led by the competition authorities, supported by normative modifications to which the different competition authorities have been adapting. Undoubtdly, in all this tour there raises as angular stone the promotion of the competence, question that provokes the worry and occupation so much the legislative one when dictates the Lay 1/2002, of February 12 of coordination of the competence of the State and the Autonomous Communities as for defense of competition, like of 
the own authorities that bet for the synergy with instruments of cooperation so efficient as the "Network of Cooperation of the Competent Organs as for Competition».

KEY WORDS: decentralized system, competence promotion, competence authorities, advantages, disadvantages.

RECIBIDO: 29.05.2015

ACEPTADO: 04.06.2015 